\title{
Acoustoelectronic method of solvents purity evaluation
}

\author{
M. Pasternak, K. Jasek
}

M. Pasternak, K. Jasek, "Acoustoelectronic method of solvents purity evaluation," Proc. SPIE 11442, Radioelectronic Systems Conference 2019, 114420T (11 February 2020); doi: 10.1117/12.2564715

SPIE. Event: Radioelectronic Systems Conference 2019, 2019, Jachranka, Poland 


\title{
Acoustoelectronic method of solvents purity evaluation
}

\author{
M. Pasternak ${ }^{\mathrm{a}}$, K. Jasek ${ }^{\mathrm{b}}$ \\ $a^{a *}$ Faculty of Electronics; ${ }^{b}$ Faculty of Advanced Technologies and Chemistry, \\ Military University of Technology, 2 Gen. S. Kaliskiego str., 00-908 Warsaw, Poland
}

\begin{abstract}
Solvents belong to an important group of special substances used in modern technological processes and in the operation of various types of systems, especially electronic ones. Relatively high consumption of these chemicals generates the need to monitor their quality and, above all, purity. There are many analytical methods for testing the purity of chemical compounds. However, they are usually time-consuming or expensive. The paper analyses the results of tests of the acoustoelectronic system designed to assess the quality of technical solvents.

The proposed method is simple, cheap and, as it seems, very effective in assessing the quality of solvents with sufficiently high evaporation rate. The method can be also applied to fast testing of light fuels. It uses the interaction between the vibrating surface of a quartz beam and the micro-droplet of the tested solvent applied to it. The theoretical basis of system operation and preliminary measurement results are also described.
\end{abstract}

Keywords: solvents, chemicals purity monitoring, loaded vibrating system, chemical sensors

\section{INTRODUCTION}

Solvents belong to a group of special industrial substances, intended for production of various types of solutions of liquids and solids, and to perform extraction, washing and other operations. More than 20 subgroups of these compounds are currently known [1]. They are subject to numerous requirements, which include in the first place: purity, high volatility (low boiling point), appropriate viscosity, high chemical and physical stability, lack of reactivity, low toxicity, etc. Many of these requirements can be met while maintaining the first one, i.e. a sufficiently high level of purity. Obviously, a small admixture of impurities usually does not significantly reduce the quality of the solvent, but it happens that the content of undesirable additives is so high that it significantly deteriorates solvent's technical properties. It should be noted that the source of contamination may be the container itself, in which the solvent is stored. High purity is a very important factor in electronic and optoelectronic applications. Unwanted admixtures can have a significant impact on the quality and reliability of the systems, especially if they are very precise and subject to stringent requirements.

The large number of types of solvents offered on the market often puts the potential purchaser in the dilemma of making a rational choice. This is the more important the larger the volume one wants to buy and the more expensive the solvent is. There are a number of analytical methods and techniques that allow a very thorough examination of the chemical composition of a particular substance, but these are generally time-consuming and expensive.

In this paper the method of solvent purity assessment using electromechanical properties of a vibrating quartz beam has been proposed. It consists in observing changes in the beam's resonance parameters during evaporation of a sample of the tested solvent placed on a surface of the beam. When this sample is applied, the amplitude and frequency of the beam's vibrations decrease rapidly and even disappear. The evaporation of the solvent then gradually returns these parameters to their nominal values. If such a return does not take place, it means that contaminants that have not yet completely evaporated remain on the resonator surface. The higher their total mass, the greater the observed difference between amplitudes and frequencies. A similar role would be played by quartz-crystal microbalance, but the difference is that beam resonators with shear vibrations can achieve higher frequencies of vibrations. It has also a very small surface area that decreases evaporation time and makes the observation of the process easier. The evaporation time of the pure water droplet with a diameter of about $0.1 \mathrm{~mm}$ takes in normal environmental conditions c.a. $6 \mathrm{~s}$. This has a positive effect on the properties of the system.

*mateusz.pasternak@ wat.edu.pl; phone +48 222618392 23; wel.wat.edu.pl

Radioelectronic Systems Conference 2019, edited by Piotr Kaniewski, Jan Matuszewski, Proc. of SPIE Vol. 11442, 114420T · (c) 2020 SPIE · CCC code: 0277-786X/20/\$21 · doi: 10.1117/12.2564715 


\section{VIBRATION OF A QUARTZ BEAM LOADED WITH LIQUID}

A quartz beam can vibrate in several different ways, depending on the type of crystal cut, the direction of the electric field applied and the way it is mounted in the housing. Shear vibrations are most often used, but bending, length or torsional vibrations can also be used in specific situations. Vibrations are forced by the piezoelectric effect. For a specific beam geometry, the highest frequency is obtained with shear vibrations and the lowest with flexural vibrations.

A dozen or so crystal quartz cuts are known which have specific, desired technical properties. AT-cut is one of the most popular in the case of quartz resonators. It provides temperature frequency stability of not less than $100 \mathrm{ppm}$ in the range from $-40 \mathrm{~d} 0+85{ }^{\circ} \mathrm{C}$ [2]. Quartz beams made of plates with such cuts work based on shear vibrations. The deformations of the beam vibrating in this way are illustrated in Fig. 1.

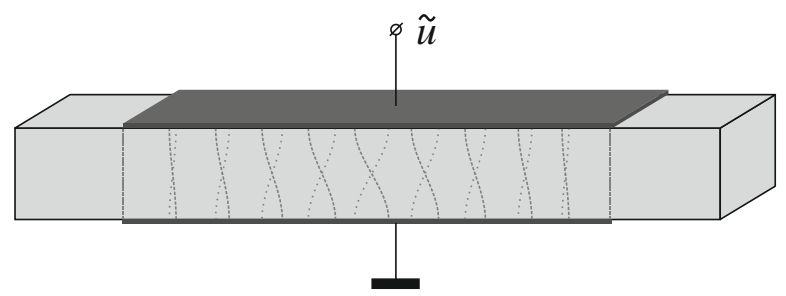

Figure 1. A beam working with shear vibrations at the basic resonance frequency. The method of beam deformation is marked with dashed lines. In order to make it visible, the amplitudes were significantly enlarged. In reality they reach values of the order of $\mathrm{nm}$.

As shown in Fig. 1. the largest amplitudes of displacements occur under metal electrodes in their central part. The maximum amplitude is reached at the fundamental resonant frequency. In addition to the frequency, this type of vibration also includes odd harmonic frequencies: 3, 5 and 7 are of technical importance - higher ones are rarely used due to insufficient amplitude of displacements. It should be noted that in this type of vibration, the way the ends of the beam are fastened has little influence on the frequency achieved.

Vibration analyses of such a system, assuming homogeneity of beam material and linear range of its deformations have been presented in numerous publications [e.g. 3, 4]. In general, they analyse the vibrations of free quartz beams, supported on one or both ends or rigidly fixed, usually without taking into consideration the mass of electrodes and/or electrical loads. However, mass loads can be introduced into the end (relationships) dependencies by changing the thickness and average density of the beam, and electrical loads by taking into account the value of the electro-mechanical coupling factor. The coordinate system adopted here is shown in Fig. 2.

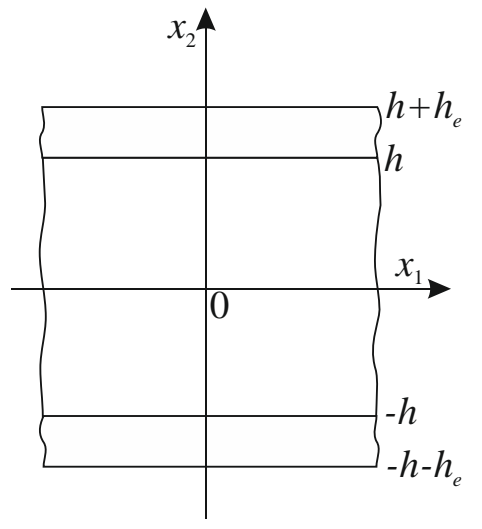

Figure. 2. The adopted coordinate system.

Assuming the symmetry of perfectly conductive electrodes with respect to $\mathrm{x} 1$ and the homogeneity of the sheardeformed substrate in the linear range, the frequency of the fundamental resonance is expressed as follows:

$$
f_{0}=\frac{1}{4 h} \sqrt{\frac{c_{66}}{\rho}}\left(1-\frac{4}{\pi^{2}} k_{26}^{2}-R\right),
$$


where $2 h$ is the beam thickness, $c_{66}$ is the shear module, $\rho$ the material density of the beam, $k_{26}^{2}$ the electromechanical coupling coefficient, and $R$ is the coefficient expressing the ratio of the mass of electrodes to the mass of crystal:

$$
R=\frac{2 \rho_{e} h_{e}}{\rho h}=-\frac{\Delta f}{f_{0}}
$$

where $\rho_{e}$ and $h_{e}$ denote respectively the material density of the electrodes and the thickness of the electrodes.

The square root factor in (1) expresses the speed of a transverse wave in a material, which means that over a distance equal to the thickness of the beam, exactly half of the acoustic transverse standing wave (near the fundamental resonant frequency) is contained. The expression in brackets describes the change in the frequency of resonator vibrations that occurs as a result of an electrical short-circuit of the piezoelectric surface and the mass loading of the electrodes on the piezoelectric surface. Amplitude of vibrations in the centre of the system $\left(x_{1}=0\right)$ can be expressed by means of the following formula:

$$
A\left(x_{2}\right)=d_{26} E_{2} \sin \frac{\pi x_{2}}{2 h},
$$

where $d_{26}$ is a piezoelectric module in the shear direction and $E_{2}$ is a homogeneous electric field between the electrodes (edge effects are omitted). The amplitude on the surface of the electrodes will reach the following values:

$$
A\left(x_{2}\right)=d_{26} E_{2} \sin \frac{\pi\left(h+h_{e}\right)}{2 h} .
$$

Applying additional mass onto the electrodes will increase the thickness and decrease the average density of the load considered as a whole, which in turn will translate into further reduction of the frequency and amplitude of the system vibrations .

For a quartz beam with an AT cut (deviated by $351^{\circ}{ }^{\circ}$ from the Z-crystallographic axis), the material parameters have the following values $[3,5]: c_{66}=29.47 \cdot 10^{9}\left[\mathrm{~N} / \mathrm{m}^{2}\right], d_{26}=3.1 \cdot 10^{-12}[\mathrm{~m} / \mathrm{V}], k_{26}=8.8 \%, \rho=2649\left[\mathrm{~kg} / \mathrm{m}^{3}\right]$. The thickness of the electrodes generally do not exceed $1 \mu \mathrm{m}[6]$.

It should be noted that the thickness of a liquid layer that modifies the frequency and amplitude of beam vibrations is not the same as the total thickness of the sample of liquid applied to the electrodes. Only its atomic layers, which are sufficiently close to the resonator surface, are involved in the transverse vibrating motion. Their number and ultimately the effective thickness of the liquid sample affecting the vibration frequency depends on the viscosity of the liquid [7].

Analyses of the properties of quartz microwaves indicate that the decrease of vibration amplitude in such systems is determined primarily by the viscosity of the loading liquid, while the decrease of frequency is determined by its mass [7, 8]. It can be assumed that after complete evaporation of volatile fractions of solvent the damping should return to the initial value, and the observed possible change of frequency of vibrations will be determined only by the mass of impurities remaining on the surface of the resonator.

The solvent quality test will be reduced to the application of a sample in the form of a micro-drop on the resonator surface, waiting for its evaporation and evaluation of a deviation of amplitude and resonant frequency from their initial values. The permissible deviations can be determined through the calibration process using a solvent with known parameters.

This procedure can be repeated. Assuming a constant volume of samples and a homogeneous distribution of contaminants in the solvent, it can be assumed that the mass of contaminants remaining after subsequent evaporations will increase by a constant value, which will increase the precision of the test.

\section{SELECTED MEASUREMENT RESULTS}

The method was tested using samples of ethyl alcohol and acetone. The first series was carried out with anhydrous A.R. alcohol (Analytical grade, pure for analysis) of $99.8 \%$ by volume with benzene admixture, the second with drinkable alcohol of $95 \%$ by volume with water (rectified spirit), the third series was carried out with technical acetone and the fourth with A.R. acetone. 
In the case of alcohols, the foreign admixture content shall not exceed $0.058 \%$ by volume of the sample. The percentage of admixtures in the examined lots of acetone was not known.

To measure the transmission parameters of the resonator, a vector network analyzer was used. In this role, however, a much simpler, dedicated measuring system can be applied. The transmission parameters of the HC-49/U-S resonator were measured. Its fundamental vibration frequency is $13.560 \mathrm{MHz}$. The diagram of the design of this resonator is shown in Fig. 3.
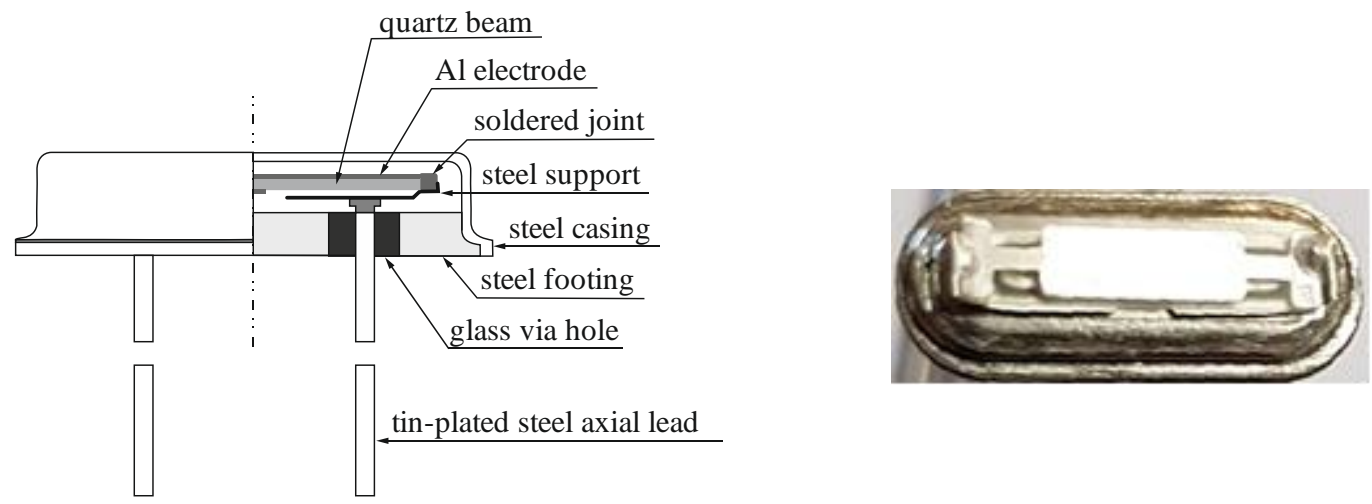

Figure 3. The structure of the HC-49U-S resonator and a photograph of this device after cutting off the upper part of the casing. The length, width and height of the housing are $11.5 ; 3.8 ; 3.69[\mathrm{~mm}]$ respectively. The size of the quartz beam depends on the resonance frequency. In the tested specimen they were as follows (length/width/thickness): 8.8/2.0/0.2 $[\mathrm{mm}]$ (the beam is transparent and for this reason only metal electrodes embedded in the walls of the beam are visible on the photograph).

Samples of $2 \pm 0.2 \mu \mathrm{l}$ of the test fluid were applied with a Hamilton 700-series microsyringe. After the application of the samples, the resonator transmission parameters were measured at minute intervals until the test substance completely evaporated. After the measurement cycle, the resonator and the microsyringe were washed with $99.8 \%$ anhydrous alcohol. Because washing has a certain influence on the surface condition of the resonator, its characteristics each time changed. It is not important, however, because at the beginning of each measurement cycle the relative change of frequency and attenuation in relation to the characteristics was measured. Fig. 4 shows an example results of transmission losses $(T L)$ measurements in the function of frequency for $99.8 \%$ anhydrous alcohol.

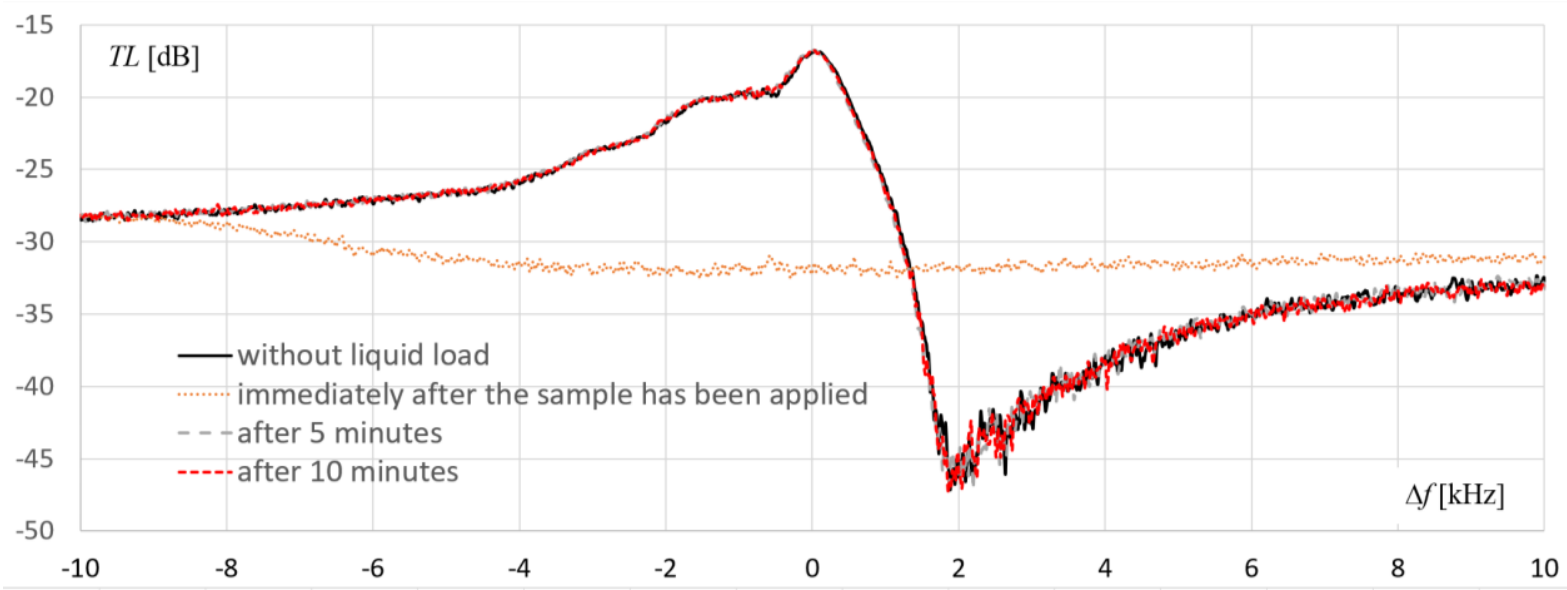

Fig. 4: Transmission losses as a function of frequency change for the resonator during evaporation of the measured $99.8 \%$ ethanol sample

As can be seen in the diagram in Fig. 4, the application of the sample caused a strong attenuation of the characteristics (practically disappearance of vibrations), but after a few minutes it completely returned to its original shape. No deviations are indicative of high purity of the sample. For this reason, this reagent was used to rinse the microsyringe and resonator during the tests. 
An example of a test result for a 95\% alcohol sample is shown in Fig. 5. Due to the water content, the resonator was returning to its original damping value slightly more slowly. However, the permanent frequency deviation of $450 \mathrm{~Hz}$ remaining after evaporation indicates a certain content of impurities.

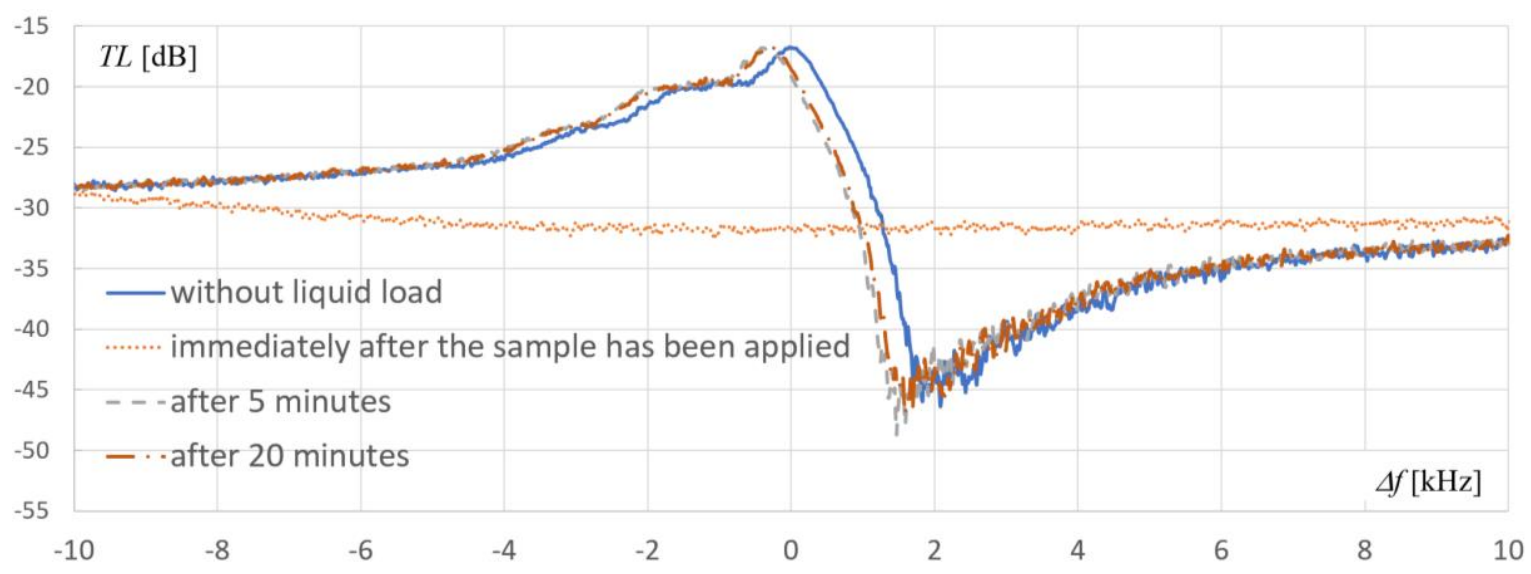

Figure 5. Transmission losses as a function of frequency change for the resonator during evaporation of the measured $95 \%$ ethanol sample.

In this case, it can be assumed that a deviation is acceptable, but higher values could be a basis for disqualifying a given lot of alcohol.

Quite unexpected results were obtained for A.R. acetone (Fig. 6.), which contained a relatively large amount of particulate matter. The recorded difference in frequency was about $700 \mathrm{~Hz}$.

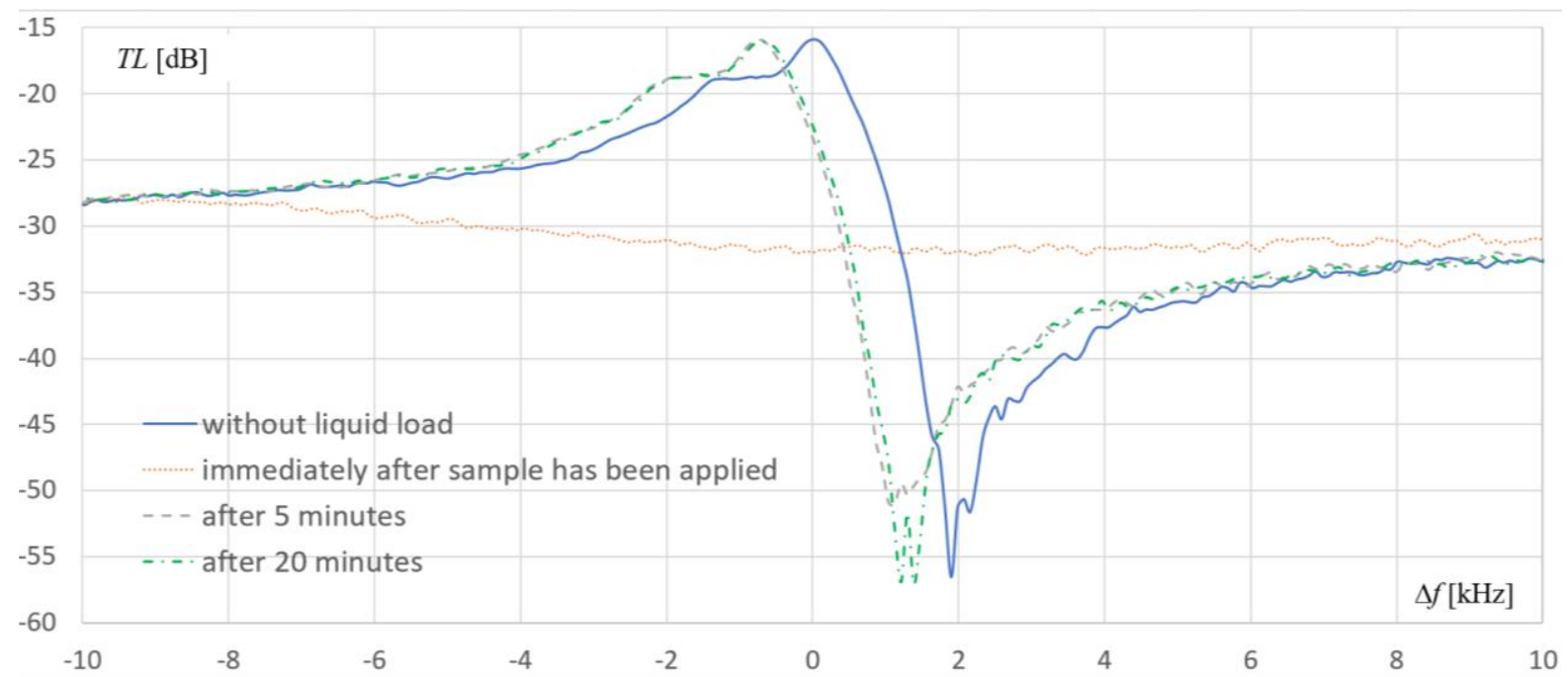

Figure 6. Transmission losses as a function of frequency change for the resonator during the evaporation of a sample of A.R. acetone

Surprisingly slightly higher contamination was noted for technical grade acetone, as illustrated in Fig. 7. 


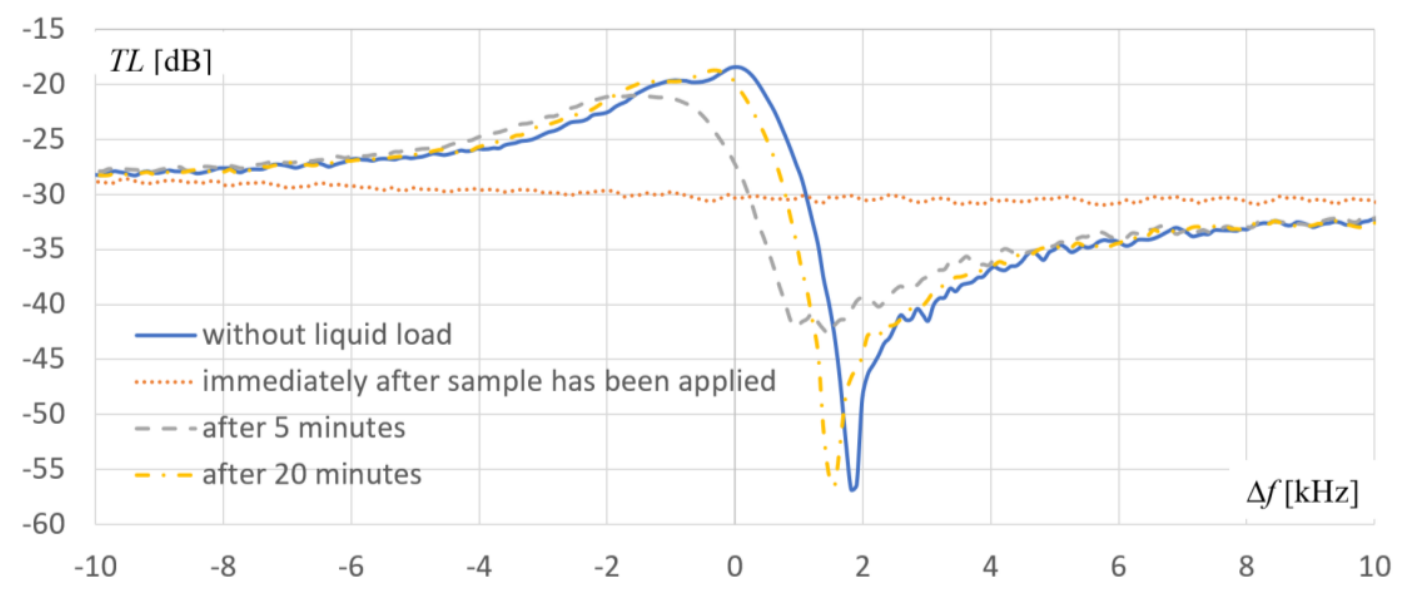

Figure 7. Transmission losses as a function of frequency for the resonator during evaporation of a sample of technical grade acetone.

Although the observed frequency deviation was only $520 \mathrm{~Hz}$, the much weaker evaporation rate and the attenuation remaining after 20 minutes at the level of about $0.6 \mathrm{~dB}$ indicates a significant admixture of liquids of low volatility. Such a reagent would not be suitable e.g. for cleaning precise optical systems.

\section{CONCLUSIONS}

The presented method of solvent purity assessment can be used to quickly estimate the content of impurities in a given solvent and their nature (solid or liquid). Its main advantage is its speed, simplicity and low price. The tests carried out convince us that in principle, after waiting about 5 minutes, it is possible to determine with certainty whether a given solvent sample is sufficiently clean for specific applications or whether a given lot of solvent does not differ in purity from the previous lot. The method can be also applied to fast testing of petrols. Since the operating frequencies of the system are relatively low, the whole device can easily be contained in a small enclosure containing a chamber with a replaceable resonator, a microcontroller chip with an analogue-to-digital converter and a small display. The main challenge with this method is to ensure that the sample volume and physical environment are sufficiently reproducible to allow for in situ testing.

\section{REFERENCES}

[1] Wypych, G., [Handbook of solvents $2^{\text {nd }}$ ed. Vol. 1. Properties], ChemTec Publ., (2014).

[2] Lam C. S., "A review of the recent development of temperature stable cuts of quartz for SAW applications," Fourth International Symposium on Acoustic Wave Devices for Future Mobile Communication Systems, 1-7 (2010).

[3] Liu N., [Vibrations of crystal plates with surface structures for resonator and sensor applications], University of Nebraska, (2012).

[4] Jang J., [Analysis of piezoelectric devices], World Scientific, 43-72 (2006).

[5] Arnau A., [Piezoelectric transducers and applications], Springer-Verlag, Berlin Heidelberg GmbH, 1-37 (2008).

[6] Khraiche M. L., Rogul J., Muthuswamy J., "Design and development of microscale thickness shear mode (TSM) resonators for sensing neuronal adhesion", Frontiers in Neuroscience, 4 June 2019, https://doi.org/10.3389/fnins.2019.00518 (2 December 2019)

[7] Jasek K., Pasternak M., Grabka M., "Wyznaczanie wysokoczęstotliwościowych parametrów materiałów lepkosprężystych za pomocą rezonatorów kwarcowych", Elektronika : konstrukcje, technologie, zastosowania, 59, 11, 34-43 (2018) (in Polish).

[8] Pasternak M., [Czujniki akustyczne], Wojskowa Akademia Techniczna, Warszawa, 132-157 (2018) (in Polish). 